Research Paper

\title{
Effect of Irradiation on Tissue Penetration Depth of Doxorubicin after Pressurized Intra-Peritoneal Aerosol Chemotherapy (PIPAC) in a Novel Ex-Vivo Model
}

\author{
Veria Khosrawipour ${ }^{1,2^{*}}$, Urs Giger-Pabst ${ }^{1,2^{*}}$, Tanja Khosrawipour ${ }^{2}$, Yousef Hedayat Pour ${ }^{3}$, David \\ Diaz-Carballo, ${ }^{4}$ Eckart Förster ${ }^{5}$, Hugo Böse-Ribeiro3 ${ }^{3}$, Irenäus Anton Adamietz ${ }^{3}$, Jürgen Zieren1, Khashayar \\ Fakhrian $^{3}$ 凶 \\ 1. Department of General Surgery \& Therapy Center for Peritonealcarcinomatosis, Marien Hospital Herne, Ruhr University Bochum, Germany. \\ 2. Basic Research Laboratory, Department of Surgery, Marien Hospital Herne, Ruhr University Bochum, Germany. \\ 3. Department of Radiation Oncology, Marien Hospital Herne, Ruhr University Bochum, Germany. \\ 4. Department of Hematology \& Medical Oncology, Marien Hospital Herne, Ruhr University Bochum, Germany. \\ 5. Department of Neuroanatomy and Molecular Brain Research, Ruhr University Bochum, Germany. \\ * Veria Khosrawipour and Urs Giger-Pabst have equally contributed to this study, and both authors should be considered first author.
}

$\triangle$ Corresponding author: Khashayar Fakhrian, Department of Radiation Oncology, Marien Hospital Herne, Ruhr-University Bochum, Hölkeskampring 40, 44625 Herne, Germany. Tel: + 49(0) 23234991531 Fax: + 49(0) 23234993306 Email: khfmed@yahoo.com.

(1) Ivyspring International Publisher. Reproduction is permitted for personal, noncommercial use, provided that the article is in whole, unmodified, and properly cited. See http://ivyspring.com/terms for terms and conditions.

Received: 2015.12.16; Accepted: 2016.03.15; Published: 2016.05.07

\begin{abstract}
Background: This study was performed to assess the impact of irradiation on the tissue penetration depth of doxorubicin delivered during Pressurized Intra-Peritoneal Aerosol Chemotherapy (PIPAC).

Methods: Fresh post mortem swine peritoneum was cut into 10 proportional sections. Except for 2 control samples, all received irradiation with 1, 2, 7 and $14 \mathrm{~Gy}$, respectively. Four samples received PIPAC 15 minutes after irradiation and 4 other after 24 hours. Doxorubicin was aerosolized in an ex-vivo PIPAC model at $12 \mathrm{mmHg} / 36^{\circ} \mathrm{C}$. In-tissue doxorubicin penetration was measured using fluorescence microscopy on frozen thin sections.

Results: Doxorubicin penetration after PIPAC ( 15 minutes after irradiation) was $476 \pm 74 \mu \mathrm{m}$ for the control sample, $450 \pm 45 \mu \mathrm{m}$ after $1 \mathrm{~Gy}(\mathrm{p}>0.05), 438 \pm 29 \mu \mathrm{m}$ after $2 \mathrm{~Gy}(\mathrm{p}>0.05)$, $396 \pm 32 \mu \mathrm{m}$ after $7 \mathrm{~Gy}(p=0.005)$ and $284 \pm 57$ after $14 \mathrm{~Gy}$ irradiation $(p<0.001)$. The doxorubicin penetration after PIPAC ( 24 hours after irradiation) was $428 \pm 77 \mu \mathrm{m}$ for the control sample, $393 \pm 41 \mu \mathrm{m}$ after $1 \mathrm{~Gy}(\mathrm{p}>0.05), 379 \pm 56 \mu \mathrm{m}$ after $2 \mathrm{~Gy}(\mathrm{p}>0.05), 352 \pm 53 \mu \mathrm{m}$ after $7 \mathrm{~Gy}(\mathrm{p}=0.008)$ and $345 \pm 53$ after $14 \mathrm{~Gy}$ irradiation $(\mathrm{p}=0.001)$.

Conclusions: Higher (fractional) radiation dose might reduce the tissue penetration depth of doxorubicin in our ex-vivo model. However, irradiation with lower (fractional) radiation dose does not affect the tissue penetration negatively. Further studies are warranted to investigate if irradiation can be used safely as chemopotenting agent for patients with peritoneal metastases treated with PIPAC.
\end{abstract}

Key words: ex-vivo, penetration, Pressurized Intra-Peritoneal Aerosol Chemotherapy (PIPAC), peritoneal carcinomatosis, radiotherapy.

\section{Introduction}

Peritoneal carcinomatosis (PC) is a common manifestation of advanced digestive-tract, mesothelioma and gynecological cancers. Patients with PC of non-gynecological malignancies have a poor prognosis with an estimated median survival of 6 months, depending on their initial Peritoneal Cancer 
Index (PCI) stage [1-2]. Intra-Peritoneal Aerosol Chemotherapy (PIPAC) is a new approach for intra-peritoneal chemotherapy (IPC). Using a micropump, the drug containing solution is delivered into the abdominal cavity in the shape of micro droplets within a $12 \mathrm{mmHg}$ "therapeutic capnoperitoneum" [3-4]. Penetration depth of cytostatics after PIPAC has been reported to be 300 to $600 \mu \mathrm{m}$ deep with tissue concentration of $0.03-4.1 \mu \mathrm{mol} / \mathrm{g}$ [3-4]. In addition to patients' characteristics and extent of the macroscopic and microscopic disease, tissue penetration depth, distribution and concentration of the cytotoxic agent are factors, which might affect the result of the IPC [4-17]. In spite of improvements in the results of the treatment after IPC, outcomes of the patients with PC remain poor. Thus, a multidisciplinary approach is necessary for the management of PC. Low dose irradiation might enhance the sensitivity of peritoneal cancer tumor cells to cytotoxic agents [18-19]. Theoretically, radiation might have a chemo-sensitizing effect and lead to higher tumor cell kill after PIPAC, provided that irradiation does not negatively impact the tissue penetration depth or tissue concentration of cytotoxic agent. This study was performed to assess the impact of irradiation on penetration depth of PIPAC.

\section{Material \& Methods}

\section{Ex-vivo PIPAC model}

Since the experiments were performed in an ex-vivo model on commercially available tissue samples no approval of the local board on animal care was required. Fresh post mortem swine peritoneum was cut into 10 proportional samples $(3 \times 3 \times 0.5 \mathrm{~cm})$. Thirty minutes postmortem, except for 2 control samples, all received radiotherapy with 1, 2, 7 and 14 Gy. Four samples were treated with PIPAC 15 minutes after and 4 other samples received PIPAC 24 hours after irradiation. These were kept in a sterile container at $100 \%$ humidity for 24 hours at $4^{\circ} \mathrm{C}$, to slow down the tissue degradation. The samples were irradiated using a 6-MV photon beam delivered by an Elekta Synergy linear accelerator (Elekta Oncology Systems, Stockholm, Sweden). Doxorubicin was aerosolized in an ex-vivo PIPAC model at $12 \mathrm{mmHg}$ $\mathrm{CO}_{2}$ at $36{ }^{\circ} \mathrm{C}$. In the center of the top cover of the PIPAC chamber, a PIPAC micro-pump was installed at a distance of $8 \mathrm{~cm}$ to the bottom. Tissue specimens were placed in the middle of the bottom of the plastic box. In-tissue doxorubicin penetration was measured using fluorescence microscopy on frozen thin sections. All experiments were repeated at least 3 times (A total of 30 samples, 6 control samples, 12 samples treated with PIPAC 15 minutes after irradiation and 12 samples treated with PIPAC 24 hours after irradiation).

The ex-vivo PIPAC model was previously described [20]. Briefly, a commercially hermetic sealable plastic box with a total volume of 3.5 liter, mimicking the abdominal cavity, was used. In the center of the top cover of the plastic box, a 10 and 5 $\mathrm{mm}$ trocar (Kii®Balloon Blunt Tip System, Applied Medical, Rancho Santa Margarita, CA, USA) were placed. Using two trocars, the nozzle of the micropump (MIP®, Reger Medizintechnik, Rottweil, Germany) and a temperature/humidity sensor (XA 1000, Lufft Mess- und Regeltechnik GmbH, Fellbach, Germany) probe were introduced. The plastic box was situated in a water bath (Typ 3043, Köttermann, Häningsen, Germany) and kept at constant temperature of $36^{\circ}$ degrees Celsius during the whole procedure (Figure 1).

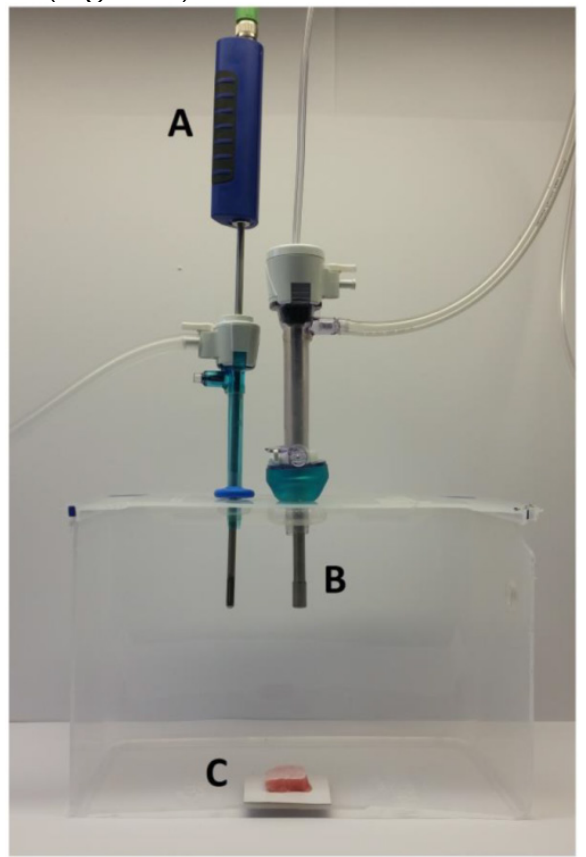

Figure 1: Laparoscopy-like ex vivo experiment with fresh swine peritoneum to investigate the spatial distribution pattern of aerosolized doxorubicin during pressurized intraperitoneal aerosol chemotherapy therapy. A: Temperature and humidity probe. B: Micropump. C: Peritoneum of the swine.

The tissue specimen of peritoneum (from German land race pigs), each measuring $3.0 \times 3.0 \times$ $0.5 \mathrm{~cm}$, were placed at the bottom of the plastic box in direct extension of the axis of the micropump nozzle in the core of the aerosol jet. The distance between the nozzle of the MIP® and the bottom of the plastic box was eight $\mathrm{cm}$. The plastic box was then tightly sealed and a constant $\mathrm{CO}_{2}$ capnoperitoneum of $12 \mathrm{~mm} \mathrm{Hg}$ (Olympus UHI-3, Olympus medical life science and industrial divisions, Olympus Australia, Notting Hill, Australia) was applied throughout the whole PIPAC 
procedure. $\quad 3 \mathrm{mg}$ Doxorubicin (Doxorubicin hydrochlorid, purchased from Teva ${ }^{\circledR}$ Pharmachemie B.V., Haarlem, Netherlands), was diluted in $50 \mathrm{ml}$ $\mathrm{NaCl} 0.9 \%$ at room temperature $\left(23^{\circ} \mathrm{C}\right)$ was aerosolized with a flow rate of $30 \mathrm{ml} / \mathrm{min}$. After the aerosol phase, the tissue specimens were exposed for another 30 minutes to aerosolized doxorubicin (exposure phase). The procedure was repeated three times.

\section{Detection of doxorubicin penetration using fluorescence microscopy}

All tissue samples were rinsed with sterile $\mathrm{NaCl}$ $0.9 \%$ solution in order to eliminate superficial cytostatics and immediately frozen in liquid nitrogen. Cryo sections $(10 \mu \mathrm{m})$ were prepared from 10 different areas of each specimen. Sections were mounted with VectaShield containing $1.5 \quad \mu \mathrm{g} / \mathrm{ml}$ 4',6-diamidino-2-phenylindole (DAPI) to stain nuclei. Penetration depth of doxorubicin was monitored using a Leica TCS SP8 confocal laser scanning microscope. The distance between the luminal surface and the innermost positive staining for doxorubicin accumulation was measured and reported in micrometers.

\section{Statistical analyses}

Experiments were independently performed three times. A total of ten tissue sections per tissue sample were subjected to doxorubicin penetration measurement. The statistical analyses were performed using Sigma Plot 12 (Systat Software Inc., California, USA). The Kruskal-Wallis One Way Analysis of Variance on Ranks was used to compare independent groups. A significant $\mathrm{p}$ - value was considered in case of $p<0.01$.

\section{Results}

Immediately after the start of the aerosol phase, the humidity reached $100 \%$ and remained constant during the whole procedure. However, the temperature in the plastic box dropped to $34^{\circ} \mathrm{C}$ at the end of the aerosol phase and climbed to $36^{\circ} \mathrm{C}$ early during the exposure phase and then remained stable until the end of the experiments. At the end of the PIPAC procedure the tissue specimens were taken out from the plastic box and microscopic evaluation was performed.

The doxorubicin penetration after PIPAC (15 minutes after irradiation) was $476 \pm 74 \mu \mathrm{m}$ for the control sample without irradiation, $450 \pm 45 \mu \mathrm{m}$ after 1 Gy $(p>0.05), \quad 438 \pm 29 \mu \mathrm{m}$ after 2 Gy $(p>0.05)$, $396 \pm 32 \mu \mathrm{m}$ after $7 \mathrm{~Gy}(\mathrm{p}=0.005)$ and $284 \pm 57$ after $14 \mathrm{~Gy}$ irradiation $(\mathrm{p}<0.001)$. Doxorubicin penetration after PIPAC (24 hours after irradiation) was
$428 \pm 77 \mu \mathrm{m}$ for the control sample without irradiation, $393 \pm 41 \mu \mathrm{m}$ after $1 \mathrm{~Gy}(\mathrm{p}>0.05), 379 \pm 56 \mu \mathrm{m}$ after $2 \mathrm{~Gy}$ $(\mathrm{p}>0.05), \quad 352 \pm 53 \mu \mathrm{m}$ after $7 \mathrm{~Gy} \quad(\mathrm{p}=0.008)$ and $345 \pm 53$ after $14 \mathrm{~Gy}$ irradiation $(\mathrm{p}=0.001)$ (Figure 2-4).

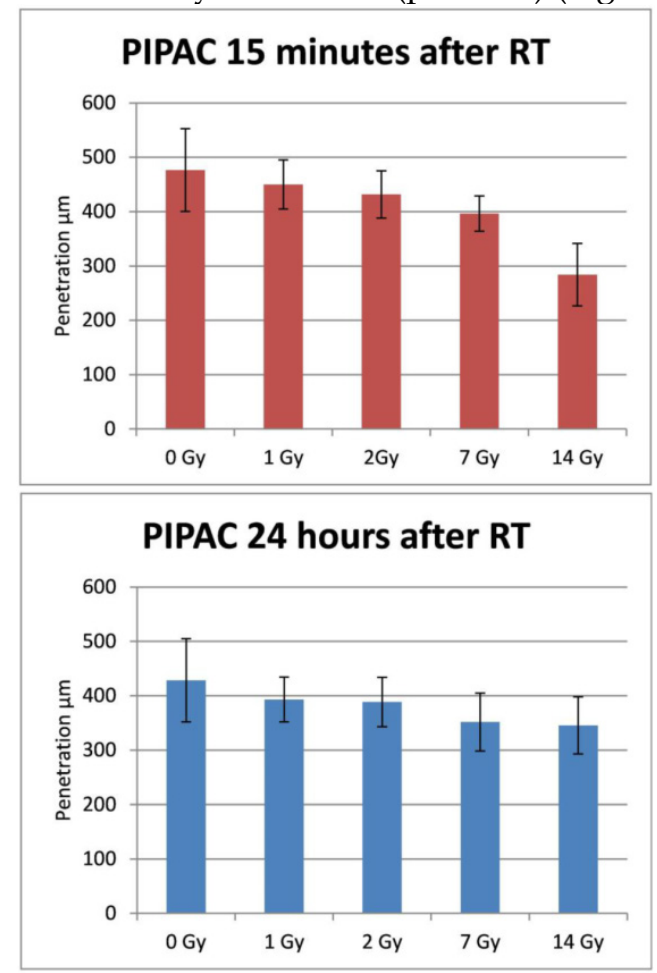

Figure 2. Tissue penetration depth of doxorubicin after irradiation followed by PIPAC after 15 minutes (top) and PIPAC after 24 hours (down). X-axis: tissue specimens in the ex-vivo PIPAC model treated right after radiation with $0,1,2,7$, and $14 \mathrm{~Gy}$. Y-axis: Penetration depth in $\mu \mathrm{m}$. RT: radiotherapy.
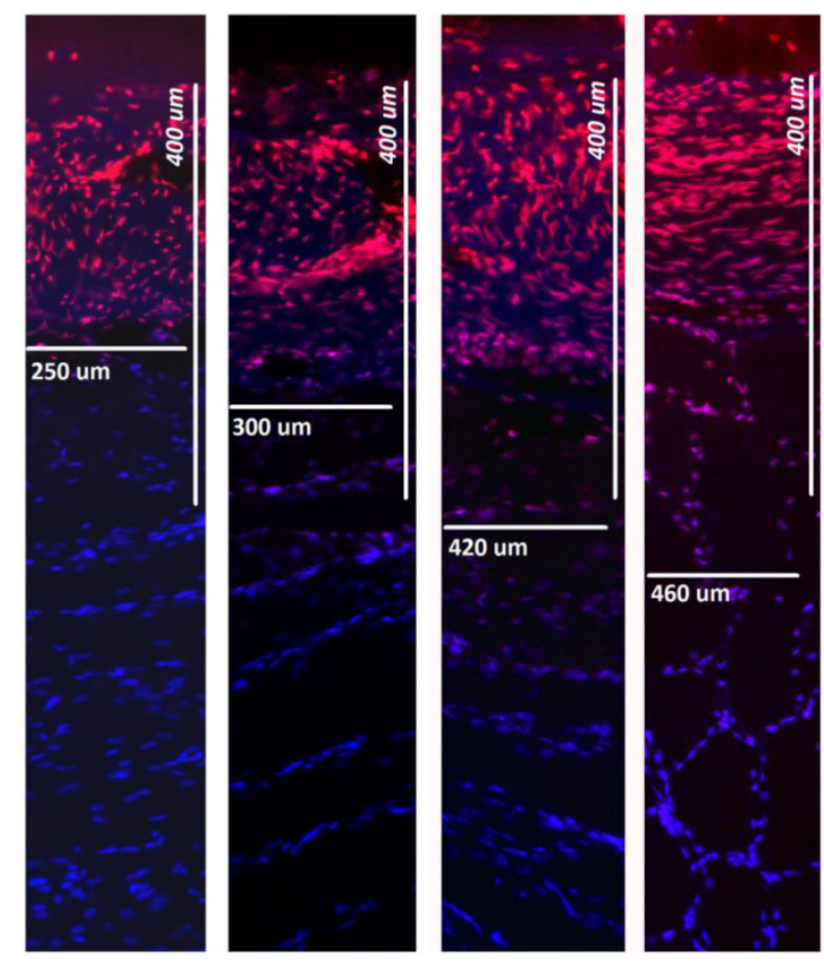

Figure 3. Fluorescence microscopic analysis of penetration depth of doxorubicin into fresh peritoneal tissue samples. Nuclei (blue) were stained with 4',6-diamidino-2-phenylindole (DAPI). Right side to left: 0, 1, 7, 14 Gy. 


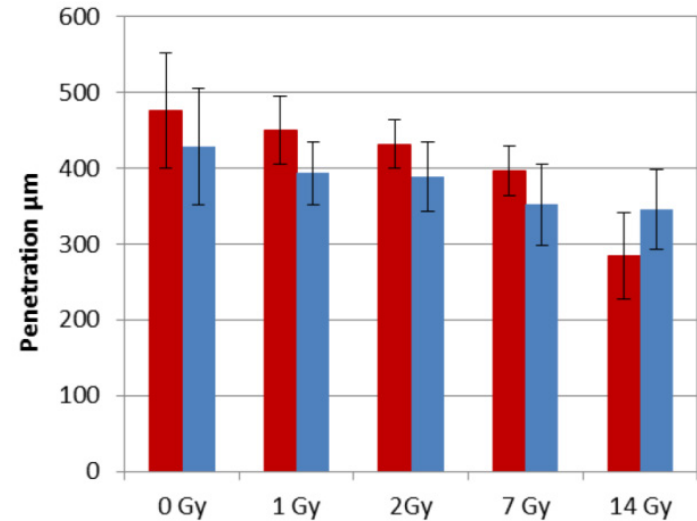

Figure 4. Comparison of penetration depth between PIPAC $15 \mathrm{~min}$ after irradiation (red) and PIPAC 24 hours after irradiation (blue).

\section{Discussion}

IPC delivered as a pressurized aerosol has been introduced as a new and innovative approach to improve the treatment of advanced PC. Animal experiments performed ex-vivo demonstrated that the infiltration of doxorubicin can be up to $500-600 \mu \mathrm{m}$ in to the peritoneal fatty tissue $[4,20]$. In order to conceive a clinical study for chemosensitizing radiotherapy before PIPAC we examined the effect of radiation on tissue penetration prior to PIPAC. The doxorubicin penetration was significantly reduced with higher radiation doses, especially after 14Gy. Interestingly, the effect of irradiation with $14 \mathrm{~Gy}$ on drug penetration was lower if the PIPAC was performed $24 \mathrm{~h}$ after irradiation compared to PIPAC 15 minutes after irradiation. The radiobiological mechanisms of this phenomenon are not addressed at the time. However, the biologic effective dose [BED = nd $(1+d / \alpha / \beta)$ ] [21] in peritoneal tissue $(\alpha / \beta=3)$ after one fraction of $14 \mathrm{~Gy}$ is $79,33 \mathrm{~Gy}$. Thus, the DNA damage at 14 Gy is more pronounceable at this dose compared to lower radiation doses. The increases doxorubicin penetration after $24 \mathrm{~h}$ post-irradiation is probably due to the (still functioning) repair mechanisms, which lead to some normalization of tissue structure and higher doxorubicin uptake in the cells. However, the low radiation dose did not show any significant reduction of doxorubicin penetration. To our knowledge, this is the first study that reports the effect of irradiation on postmortem swine peritoneum in combination with PIPAC. Furthermore, this is also the first study that uses postmortem peritoneal tissue for radiobiological analysis and reports an influence of radiation on the peritoneal tissue penetration. However, the biological mechanisms underlying principles of the observed and radiation-induced changes are currently unclear. Moreover, it is known that depending on the tissue type, postmortem changes most likely include metabolic, physical and structural as well as biochemical changes [22]. These alterations, if additionally affected by the exposure to ionizing radiation, may influence the ability of the applied Doxorubicin aerosol to penetrate into the investigated postmortem peritoneal tissue. Detailed studies on the processes that underline the observed changes in tissue penetration due to radiation exposure of postmortem tissue are currently designed. The results of our study should be interpreted with caution. Firstly, our experiments were performed in an ex-vivo postmortem model. Thus, although peritoneum is not a shock organ like heart, brain or liver, its response to irradiation in a living organism with regular blood circulation might be totally different. Secondly, clinical data about efficacy and toxicity of chemopotenting radiotherapy for the systemic treatment of peritoneal cancer is rare, but in fact there is no data available on chemopotenting radiotherapy prior to or after IPC. Nevertheless, the described findings might have important implications with respect to the practice of PIPAC therapy.

\section{Conclusions}

Higher (fractional) radiation dose might reduce the penetration depth of doxorubicin in our ex-vivo model. However, irradiation with lower (fractional) radiation dose does not affect the tissue penetration negatively. Further experimental ex-vivo and in-vivo as well as clinical studies are warranted to investigate if irradiation can be used as chemopotenting agent for patients with peritoneal metastases treated with PIPAC without reducing the penetration depth of cytotoxic agent or increasing the toxicity.

\section{Author Contribution}

Veria Khosrawipour: Study design, laboratory analysis, data acquisition and drafting of the manuscript.

Urs Giger-Pabst: Study design, supervision of the study, data interpretation, drafting and critical revision for important intellectual content of the manuscript.

Tanja Khosrawipour: Laboratory analysis, data acquisition and manuscript drafting

Yousef Hedayat Pour: Laboratory analysis, data acquisition and manuscript drafting

David Diaz-Carballo: Supervision of the experiments, statistical analysis, data interpretation and manuscript drafting.

Eckart Förster: Laboratory analysis, data acquisition and drafting of the manuscript

Hugo Böse-Ribeiro: Laboratory analysis, data acquisition and drafting of the manuscript 
Irenäus Anton Adamietz: Critical revision for important intellectual content of the manuscript.

Jürgen Zieren: Drafting and critical revision for important intellectual content of the manuscript.

Khashayar Fakhrian: Supervision of the study, drafting and critical revision for important intellectual content of the manuscript.

\section{Competing Interests}

This study was financed by institutional funds. The authors have no conflicts of interest or financial ties to disclose.

\section{References}

1. Sadeghi M, Arvieux C, Glehen $\mathrm{O}$, et al. Peritoneal Carcinomatosis from Non-Gynecological Malignancies: Results of the EVOCAPE 1 Multicentric Prospective Study. Cancer 2000;88:358-363.

2. Solaß W, Hetzel A, Nadiradze G, et al. Description of a novel approach for intraperitoneal drug delivery and the related device. Surg Endosc 2012;26:1849-1855.

3. Reymond MA, Solaß W. Pressurized Intraperitoneal Aerosol Chemotherapy Cancer under Pressure (1st ed). Berlin: De Gruyter; 2014

4. Solaß W; Kerb R, Mürdter T, et al. Intraperitoneal chemotherapy of peritoneal carcinomatosis using pressurized aerosol as an alternative to liquid solution: First evidence for efficacy. Ann Surg Oncol 2014;21:553-559.

5. Jacquet P, Stuart OA, Chang D, et al. Effects of intra-abdominal pressure on pharmacokinetics and tissue distribution of doxorubicin after intraperitoneal administration. Anticancer Drugs 1996;7:596-603.

6. Sugarbaker PH. Peritoneal carcinomatosis: Is cure an option? J Clin Oncol 2003;21:762-764.

7. Verwaal VJ, van Ruth S, de Bree E, et al. Randomized trial of cytoreduction and hyperthermic intraperitoneal chemotherapy with peritoneal carcinomatosis of colorectal cancer. J Clin Oncol 2003;21:3737-3742.

8. Glehen $\mathrm{O}$, Kwiatkowski $\mathrm{F}$, Sugarbaker $\mathrm{PH}$, et al. Cytoreductive surgery combined with perioperative intraperitoneal chemotherapy for the management of peritoneal carcinomatosis from colorectal cancer: A multi-institutional study. J Clin Oncol 2004;22:3284-3292.

9. Elias D, Gilly F, Boutitie F, et al. Peritoneal colorectal carcinomatosis treated with surgery and perioperative intraperitoneal hyperthermic chemotherapy: Retrospective analysis of 523 patients from a multicentric French study. J Clin Oncol 2010;28:63-68.

10. Verwaal VJ, Bruin S, Boot $\mathrm{H}$, et al. 8-year follow-up of randomized trial: Cytoreduction and hyperthermic intraperitoneal chemotherapy versus systemic chemotherapy in patients with peritoneal carcinomatosis of colorectal cancer. Ann Surg Oncol 2008;15:2426-2432.

11. Dedrick RL, Myers CE, Bungay PM, et al. Pharmacokinetic rational for the peritoneal drug administration in the treatment of ovarian cancer. Cancer Treat Rep 1978;6:1-11.

12. Los G, Mutsaers PH, van der Vijgh WJ, et al. Direct diffusion of cis-diamminedichloroplatinum(II) in intraperitoneal rat tumor after intraperitoneal chemotherapy: A comparison with systemic chemotherapy. Cancer Res 1989;49:3380-3384.

13. Jacquet P, Sugarbaker PH. Peritoneal-plasma barrier. Cancer Treat Res 1996;82:53-63.

14. Jain RK. Barriers to drug delivery in solid tumors. Sci Am. 1994;271:58-65

15. Flessner MF. The transport barrier in intraperitoneal therapy. Am J Physiol Renal Physiol 2005;288:433-442.

16. Rossi CR, Mocellin S, Pilati P, et al. Pharmacokinetics of intraperitoneal cisplatin and doxorubicin. Surg Oncol Clin N Am 2003;12(3):781-94.

17. Jacquet $\mathrm{P}$, Stuart OA, Chang D, Sugarbaker PH. Effects of intra-abdominal pressure on pharmacokinetics and tissue distribution of doxorubicin after intraperitoneal administration. Anticancer Drugs 1996;7(5):596-603.

18. Kunos CA, Sill MW, Buekers TE, et al. Low dose abdominal radiation as a docetaxel chemosensitizer for recurrent epithelial ovarian cancer: A phase I study of the Gynecologic Oncology Group. Gynecol Oncol 2011;120(2):224-228.

19. Regine WF, Hanna N, Garofalo MC, et al. Low-dose radiotherapy as a chemopotentiator of gemcitabine in tumors of the pancreas or small bowel: A phase I study exploring a new treatment paradigm. Int J Radiation Oncology Biol Phys 2007,68(1):172-177.

20. Solaß W, Herbette A, Schwarz T, et al. Therapeutic approach of human peritoneal carcinomatosis with Dbait in combination with capnoperitoneum: proof of concept. J Surg Endosc 2012;26:847-852.

21. Lee SP, Leu MY, Smathers JB, et al. Biologically effective dose distribution based on the linear quadratic model and its clinical relevance. Int J Radiat Oncol Biol Phys. 1995 Sep 30;33(2):375-89.
22. Chagnot C, Ve nien A, Jamme F, et al. Hyperspectral deep ultraviolet autofluorescence of muscle fibers is affected by postmortem changes. J Agric Food Chem 2015;63(19):4782-4789. 